\title{
Pode o peso ao nascer influenciar o estado nutricional, os níveis de atividade física e a aptidão física relacionada à saúde de crianças e jovens?
}

\author{
Can birth weight influence nutritional status, \\ physical activity levels and health-related \\ physical fitness levels of children \\ and adolescents?
}

João Wellington Oliveira BARROS'

Marcelus Brito de ALMEIDA' ${ }^{1}$

Marcos André Moura dos SANTOS²

Paulo Roberto de SANTANA ${ }^{1}$

Florisbela de Arruda Câmara e Siqueira CAMPOS1

Carol Góis LEANDRO1

RE S U M O

A aptidão física relacionada à saúde é definida como um conjunto de atributos fisiológicos que o indivíduo apresenta para o desempenho de atividades físicas diárias sem fadiga excessiva. Sabe-se que fatores como atividade física e estado nutricional influenciam diretamente os níveis de aptidão física. Entretanto, o aporte adequado de nutrientes nos períodos iniciais da vida é determinante para o crescimento e o desenvolvimento de órgãos e de sistemas. O peso ao nascer está relacionado ao desenvolvimento intrauterino bem como à prevalência de doenças cardiovasculares e metabólicas. Recentemente o peso ao nascer tem sido associado aos níveis de aptidão física relacionada à saúde. O mecanismo subjacente pode estar relacionado aos efeitos decorrentes de insultos ocorridos no período crítico do desenvolvimento, com alterações no padrão de eventos celulares. As consequências estão na aquisição de padrões fisiológicos maduros do organismo e na ocorrência de eventos metabólicos, com prejuízo na aptidão física. O presente estudo propõe uma análise sobre a aptidão física relacionada à saúde e sua relação com a atividade física, o estado nutricional e com o peso ao nascer do indivíduo. Os termos de indexação utilizados foram: physical fitness, programming, physical activity, nutritional

\footnotetext{
${ }^{1}$ Universidade Federal de Pernambuco, Centro Acadêmico de Vitória, Núcleo de Educação Física e Ciências do Esporte. R. Alto do Reservatório, s/n., Bela Vista, 55608-680, Vitória de Santo Antão, PE, Brasil. Correspondência para/ Correspondence to: J.W.O. BARROS.E-mail: <jwobarros@yahoo.com.br>.

2 Universidade Federal de Pernambuco, Centro de Ciências da Saúde, Departamento de Nutrição. Recife, PE, Brasil.
} 
status e low birth weight. Concluiu-se que indivíduos nascidos com baixo peso apresentam alterações no estado nutricional com consequências negativas para a atividade física e aptidão física relacionada à saúde.

Termos de indexação: Atividade física. Baixo peso ao nascer. Estado nutricional. Programação.

\section{A B S T R A C T}

Heath-related physical fitness is defined as a set of physiological attributes that are necessary for performing daily physical activities without excessive fatigue. Physical activity and nutritional status can influence the individual's physical fitness levels directly. However, the appropriate supply of nutrients in early life is crucial for the growth and development of organs and systems. Birth weight is associated with intrauterine development and to the incidence of cardiovascular and metabolic diseases. Recently, birth weight has been associated with health-related physical fitness levels. The underlying mechanism may be related to the effects of insults that occur during a critical period of human development, with changes in the standard cellular events. The acquisition of mature physiological body patterns and the occurrence of metabolic events are affected, resulting in impaired physical fitness. The present study proposes an analysis on physical fitness, nutritional status and birth weight. The keywords used were: physical fitness, programming, physical activity, nutritional status and low birth weight. In conclusion, low-birth weight individuals present changes in the nutritional status which have a negative impact on physical activity and health-related physical fitness.

Indexing terms: Motor activity. Infant, low birth whight. Nutritional status. Programming.

\section{N T R O D U ÇÃ O}

A aptidão física é definida como a capacidade para desempenhar atividades físicas diárias sem fadiga excessiva e está associada com o baixo risco de desenvolvimento de doenças hipocinéticas ${ }^{1}$. Os componentes da aptidão física são a aptidão cardiorrespiratória, a força muscular, a resistência muscular, a flexibilidade e a composição corporal².

Estudos sobre aptidão física têm procurado analisar sua relação com os riscos para o desenvolvimento de doenças crônicas ${ }^{3,4}$. A aptidão cardiorrespiratória, por exemplo, tem sido associada à menor predisposição a doenças cardiovasculares $^{3,5}$ e metabólicas ${ }^{6}$. A associação entre aptidão cardiorrespiratória e mortalidade é comparável a outros fatores de risco à saúde, como excesso de peso corporal, pressão sanguínea, colesterol e fumo ${ }^{7-9}$. Nessa perspectiva, um estudo realizado com adolescentes (12-19 anos de idade) e adultos (20-49 anos de idade) revelou que altos níveis de aptidão cardiorrespiratória estão relacionados à redução de riscos de doenças cardiovasculares ${ }^{10}$. Neste estudo, a aptidão cardiorrespiratória foi determinada em teste de esteira rolante por $6 \mathrm{mi}$ nutos (caminhada ou corrida) por meio da medida direta de $\mathrm{VO}_{2 \text { Máx' }}$ que é comumente empregada como critério de medida para aferição da aptidão cardiorrespiratória e consiste no volume máximo de oxigênio que um indivíduo pode consumir e utilizar durante uma atividade física de intensidade progressiva'.

Outro estudo procurou investigar a relação entre aptidão cardiorrespiratória e composição corporal em crianças dos 7 aos 10 anos de idade. Foram coletados o peso, a altura, a circunferência da cintura e o Índice de Massa Corporal (IMC). A aptidão cardiorrespiratória foi realizada pelo teste de corrida de 20 metros. Os resultados obtidos apontaram que a composição corporal é inversamente relacionada à aptidão cardiorrespiratória ${ }^{11}$. Outros benefícios, como o aumento da sensibilidade à insulina, também foram observados com a melhora da aptidão cardiorrespiratória ${ }^{12}$.

No âmbito da força e da resistência musculares, pesquisas mostram que o desenvolvimento desses componentes, além de proteger contra doenças cardíacas, proporciona uma maior formação óssea ${ }^{5,13}$. Dessa forma, maiores níveis de força e resistência podem evitar ou retardar problemas como a osteopenia e a osteoporose, além de diminuir os riscos de fraturas ${ }^{13,14}$. Ademais, essa 
ação poderia ser complementada com a flexibilidade, visto que um maior grau de flexibilidade é fator importante para a saúde, pois está associado à melhora na mobilidade articular, prevenção de lesões e dores por esforço físico ${ }^{15}$.

Em contrapartida, os riscos à saúde podem ser percebidos na medida em que níveis inferiores de força e resistência musculares potencializam o desenvolvimento de doenças cardíacas ${ }^{5}$. Gale et al. ${ }^{16}$ verificaram que em adultos a diminuição da força e da resistência musculares estava associada à predisposição de doenças cardiovasculares. As medidas de força foram obtidas utilizando-se o método da dinamometria manual. Concluiu-se que a perda de massa muscular que ocorre com o avanço da idade reduz os níveis de força e resistência musculares e torna os indivíduos mais suscetíveis a problemas cardiovasculares ${ }^{16}$.

Estudos recentes têm analisado de forma direta a ligação entre aptidão física relacionada à saúde e fatores como a atividade física e o estado nutricional $\left.\right|^{4,17,18}$ e revelaram que indivíduos com sobrepeso, obesidade ou desnutridos apresentam níveis inferiores de atividade física e aptidão física quando comparados a indivíduos com peso normal. Diante do exposto, esta revisão tem como objetivos: 1) abordar a relação entre atividade física, estado nutricional e nível de aptidão física relacionada à saúde; 2) analisar a influência do peso ao nascer no nível de aptidão física relacionada à saúde de crianças e jovens.

\section{M É T O D O S}

Para a realização deste artigo, foram utilizadas as bases de dados PubMed, MedLine, Lilacs e Bireme. Os termos de indexação utilizados foram: physical fitness, programming, physical activity, nutritional status e low birth weight. A partir da obtenção e leitura dos artigos, suas referências foram rastreadas à procura de outros estudos potencialmente utilizáveis. Num total de 72 artigos pesquisados, houve a inclusão de 50 artigos de acordo com a relevância do estudo para os tópicos abordados. Esta revisão incluiu estudos clássicos sobre programação fetal e aptidão física relacionada à saúde bem como pesquisas mais recentes sobre os temas abordados.

\section{Fatores que influenciam o nível de aptidão física relacionada à saúde: atividade física e estado nutricional}

A prática de atividade física habitual está associada à melhora nos níveis de aptidão física relacionada à saúde ${ }^{19-21}$. Por atividade física compreende-se qualquer movimento do músculo esquelético que demande gasto energético ${ }^{22}$. Quanto ao nível de atividade física, o indivíduo pode ser classificado como ativo ou inativo de acordo com o dispêndio energético em atividades físicas 5 . A medida mais usada para determinar o gasto energético num esforço físico é o Equivalente Metabólico (MET) ${ }^{23}$, embora possa ser utilizada também Kcal ${ }^{5,23}$. Um MET representa o gasto energético de um indivíduo em repouso ${ }^{23}$. Dessa forma, um indivíduo é considerado ativo quando realiza atividades físicas diárias com um dispêndio energético em torno de 450-750 METs/min/semana ${ }^{5}$. Por exemplo, se um indivíduo realiza caminhadas, que correspondem a aproximadamente 3.3 MET, por 30 minutos, 5 vezes por semana, terá um dispêndio energético de $495 \mathrm{METs} / \mathrm{min} / \mathrm{semana}$ $(3.3 \times 30 \times 5)^{5}$.

De acordo com a Organização Mundial de Saúde (OMS) ${ }^{24}$, crianças e jovens podem ser beneficiados pela prática de atividades físicas, com a melhora da aptidão física e a diminuição dos riscos de aparecimento de doenças cardiovasculares e diabetes tipo II. Entretanto, para que sejam alcançados tais objetivos, o guia canadense de atividade física recomenda um mínimo de 90 minutos de atividades físicas diárias, que podem ser acumuladas em períodos de 5 a 10 minutos ao longo do $\mathrm{dia}^{25}$. Sobre esse aspecto, Huang \& Malina ${ }^{19}$ verificaram a relação entre atividade física e aptidão física em 282 adolescentes de Taiwan entre 12 e 14 anos de idade. A aptidão física foi mensurada com testes de força e resistência abdominal (curl up), flexibilidade (sit and reach) e aptidão cardior- 
respiratória (one mile test). O nível de atividade física foi determinado usando o recordatório de atividades físicas desenvolvido por Bouchard e colaboradores. Os resultados comprovaram que os adolescentes fisicamente mais ativos apresentavam melhores níveis de aptidão cardiorrespiratória, resistência muscular e flexibilidade.

Ademais, a atividade física quando associada à nutrição, além de melhorar os níveis de aptidão física, também diminui os riscos de doenças crônico-degenerativas e metabólicas, como a obesidade ${ }^{20,26,27}$. Percebe-se, dessa forma, que há uma relação entre nível de atividade física, aptidão física e estado nutricional do indivíduo $0^{17,28}$.

\section{Estado nutricional atual}

O estado nutricional está diretamente ligado à aptidão física ${ }^{21,28}$, e indivíduos que estão abaixo do peso demonstram níveis inferiores de aptidão física relacionada à saúde ${ }^{17}$. Pesquisa desenvolvida com participantes de Moçambique entre 6 e 18 anos de idade verificou a relação entre estado nutricional e aptidão física. O estado nutricional foi avaliado de acordo com os índices antropométricos peso/altura, altura/idade e peso/ idade ${ }^{29}$. Os componentes da aptidão física foram avaliados de acordo com uma bateria de 8 testes físicos: flexibilidade (sit and reach), força e resistência musculares (trunk lift, handgrip, curl up, salto horizontal e flexão de braço) e aptidão cardiorrespiratória (one mile test). Foi relatado que crianças e adolescentes desnutridos apresentam níveis inferiores de atividade física e aptidão física quando comparados a indivíduos com peso normal17.

Nessa perspectiva, estudos realizados a fim de avaliar a força muscular por meio da preensão manual indicaram que esse método pode ser um importante indicador da aptidão física e do estado nutricional, visto que a perda de massa muscular mostra baixos níveis de força ${ }^{16,30,31}$, particularmente em indivíduos com desnutrição crônica ou que estão abaixo do peso ${ }^{30}$. Estudo biomecânico realizado no nordeste brasileiro por Paiva et al. ${ }^{32}$ relatou que crianças desnutridas apresentam atrofia muscular e maior rigidez muscular quando comparadas a crianças eutróficas. Na avaliação biomecânica, foi utilizado um ergômetro de tornozelo, e o estado nutricional foi determinado de acordo com as diretrizes da OMS 29 .

Por outro lado, indivíduos com excesso de gordura corporal também apresentam níveis inferiores de aptidão física ${ }^{28,33}$. Um estudo longitudinal avaliou a relação entre excesso de peso e aptidão física em 6287 crianças e adolescentes americanos entre 5 e 14 anos de idade no período de 2001 a 2003. O estado nutricional foi determinado por meio do índice de massa corporal e a aptidão física por meio de testes de flexibilidade (sit and reach), força e resistência musculares (flexão de braço), e aptidão cardiorrespiratória (shuttle run test). Nesse estudo, os resultados apontaram que indivíduos obesos têm níveis inferiores de aptidão física relacionada à saúde ${ }^{33}$. Deforche et al. ${ }^{34}$ também mostraram essa relação ao relatar que indivíduos obesos entre 12 e 18 anos de idade apresentam menores níveis de resistência muscular e aptidão cardiorrespiratória quando comparados aos não obesos.

Outro estudo realizado na China com 6929 crianças entre 6 e 12 anos de idade investigou a associação entre o peso corporal e a aptidão física. $\mathrm{O}$ estado nutricional foi determinado por meio do IMC e dos índices antropométricos da OMS ${ }^{29}$. Foram avaliadas a aptidão cardiorrespiratória, a força muscular de membros inferiores e a velocidade por meio dos testes shuttle run, salto horizontal e corrida de $50 \mathrm{~m}$, respectivamente. Os resultados mostraram que crianças obesas apresentam menores valores de força muscular, de aptidão cardiorrespiratória e de velocidade quando comparadas a crianças com sobrepeso e com peso normal ${ }^{18}$.

No entanto, o período fetal também tem sido reconhecido como fator determinante para o nível de aptidão física ${ }^{35}$. Estudos recentes têm relatado que uma nutrição inadequada, com carência ou excesso de nutrientes em períodos críticos do desenvolvimento, pode levar ao baixo peso 
ao nascer e está atrelada a modificações no desenvolvimento ao longo da vida pós-natal ${ }^{36}$.

\section{Influência do peso ao nascer sobre os níveis de aptidão física relacionada à saúde}

O peso ao nascer tem sido associado ao desenvolvimento intrauterino bem como à prevalência de doenças cardiovasculares e metabólicas na idade adulta ${ }^{37}$. O baixo peso ao nascer é classificado de duas formas: baixo peso - entre $1500 \mathrm{~g}$ e $2499 \mathrm{~g}^{29}$ e muito baixo peso - inferior a $1500 \mathrm{~g}^{29}$. Ele pode ser resultado de uma curta gestação e/ou retardo no crescimento intrauterino ${ }^{38}$ proveniente de insultos como hormônios, drogas ou desnutrição, ocorridos no período crítico do desenvolvimento fetal ${ }^{39,40}$. O mecanismo parece estar associado aos efeitos decorrentes de insultos ocorridos no período crítico do desenvolvimento, alterando o padrão de eventos celulares, com consequências nocivas tanto na aquisição de padrões fisiológicos maduros do organismo quanto para a ocorrência de eventos metabólicos ${ }^{41}$. O fenômeno biológico subjacente a esses processos é denominado de programação ${ }^{39}$.

O termo programação é utilizado para descrever o processo pelo qual um estímulo ou insulto, quando aplicado em períodos críticos do desenvolvimento, tem efeitos permanentes sobre a estrutura e as funções do organismo ${ }^{39}$. Os estudos que envolvem programação fetal com humanos geralmente associam a carência nutricional como estímulo indutor de programação ${ }^{37,41}$. Recentemente tem sido estudadas as consequências do baixo peso ao nascer sobre o nível de aptidão física relacionada à saúde ${ }^{35,42,43}$.

Baixos níveis de aptidão física que apresentem potencial para afetar a saúde, a aprendizagem e o comportamento estão associados a crianças que nasceram com muito baixo peso ${ }^{35,42}$. Estudo realizado com crianças que nasceram com muito baixo peso revelou níveis inferiores da aptidão cardiorrespiratória e da força muscular dessas crianças $^{44}$.
Com relação à aptidão cardiorrespiratória, Smith et al. ${ }^{43}$ mostraram por meio de testes de função pulmonar, como a espirometria, e de aptidão física, como a caminhada de 6 minutos, que a capacidade aeróbia de crianças de 10 anos de idade que nasceram com muito baixo peso é a metade da de crianças nascidas com peso normal. Esses resultados podem ser explicados pelas possíveis disfunções pulmonares em crianças nascidas com muito baixo peso ${ }^{42,43}$, com redução do consumo de oxigênio ${ }^{35}$. Sobre esse aspecto, Kriemler et al. ${ }^{45}$ avaliaram a capacidade aeróbia de crianças e adolescentes entre 9 e 15 anos de idade - que apresentavam ou não doença pulmonar crônica - nascidos com muito baixo peso. Os dados revelaram que, com ou sem doença pulmonar crônica, indivíduos nascidos com muito baixo peso apresentam algum tipo de disfunção pulmonar e, consequentemente, reduzida capacidade aeróbia. Soma-se a isso o fato de crianças nascidas com baixo peso possuírem níveis inferiores de atividade física ${ }^{35}$, o que também confere níveis inferiores de aptidão cardiorrespiratória ${ }^{19}$. Problemas na capacidade aeróbia também confirmam outros achados de aumentada fatigabilidade em indivíduos que nasceram com muito baixo peso ${ }^{45}$.

Indivíduos nascidos com muito baixo peso também apresentam níveis reduzidos de força, resistência ${ }^{46}$ e flexibilidade quando comparados a indivíduos nascidos com peso normal ${ }^{42,46}$. Estudo realizado por Rogers et al. ${ }^{42}$ relatou que crianças nascidas com muito baixo peso apresentavam aos 17 anos de idade níveis inferiores de força, resistência e flexibilidade quando comparadas a crianças nascidas com peso normal. Foram realizados testes de força superior de tronco (push up), de resistência abdominal (curl up) e de flexibilidade do tronco (flexômetro), além de questionários para aferição do nível de atividade física habitual. O estudo apontou que os baixos níveis de força, resistência muscular e flexibilidade podem ser resultado de uma reduzida participação em atividades físicas. O menor grau de flexibilidade foi relacionado a uma menor participação em atividades físicas, rigidez muscular e anormalidades no tônus muscular. 
Com relação à força, bons níveis de força muscular tendem a estar associados a uma nutrição adequada nos primeiros anos de vida, e, a partir desse entendimento, o período fetal parece ser um dos fatores determinantes para o desenvolvimento da força muscular ${ }^{16}$, o que poderia explicar os achados de melhores resultados de força muscular em crianças nascidas com peso normal ${ }^{47}$.

O ambiente pré-natal exerce influência no desenvolvimento do organismo, e eventos estressantes durante a gravidez podem induzir alterações no ambiente fetal ${ }^{48}$. Tais alterações podem resultar em consequências estruturais e funcionais a curto e a longo prazo ${ }^{49}$. As consequências podem ser disfunções pulmonares ${ }^{43}$, cardiovasculares ${ }^{41}$ e alterações na estrutura e funções no músculo esquelético ${ }^{50}$, levando a um prejuízo no nível de aptidão física relacionada à saúde ${ }^{42,43}$.

\section{O N CLUS Ã O}

Indivíduos que nasceram com baixo peso apresentam níveis inferiores de aptidão física relacionada à saúde quando comparados aos nascidos com peso normal. O fato aponta possíveis alterações fisiológicas ocorridas no ambiente fetal que influenciaram a aptidão física. Outro fator agravante para esse resultado é a reduzida prática de atividades físicas por esse grupo de indivíduos $^{35,42,45}$. Soma-se ainda a esses aspectos a influência do estado nutricional: indivíduos que estão desnutridos ou com excesso de peso apresentam deficits de desempenho quando comparados a indivíduos eutróficos, com prejuízo direto no nível de aptidão física relacionada à saúde.

\section{COLABORADORES}

J.W.O. BARROS realizou a pesquisa de todos os blocos de conteúdos, a estruturação, a redação e a edição final do manuscrito. M.B. ALMEIDA e M.A.M. SANTOS participaram da pesquisa, da análise e da seleção de artigos sobre o conteúdo relacionado à atividade física. P.R. SANTANA participou da pesquisa, da análise e da seleção de artigos sobre o conteúdo relacionado aos aspectos nutricionais e ao peso ao nascer. F.A.C.S. CAMPOS participou da pesquisa, da análise e da seleção de artigos sobre o conteúdo relacionado aos aspectos nutricionais. C.G. LEANDRO participou da orientação, da estruturação e da edição final do artigo.

\section{REFER Ê NCIAS}

1. Wilder RP, Greene JA, Winters KL, Long WB, Gubler K, Edlich RF. Physical fitness assessment: an update. J Long Term Eff Med Implants. 2006; 16(2):193-204. doi: 5411b78b6ac8ee0f,21dd375d517fa227.

2. Douris $P$, Chinan A, Gomez M, Aw A, Steffens D, Weiss $S$. Fitness levels of middle aged martial art practitioners. Br J Sports Med. 2004; 38(2):143-7. doi: 10.1136/bjsm.2002.001768.

3. Church TS, LaMonte MJ, Barlow CE, Blair SN. Cardiorespiratory fitness and body mass index as predictors of cardiovascular disease mortality among men with diabetes. Arch Intern Med. 2005; 165(18):2114-20. doi: 165/18/211410.1001/ archinte.165.18.2114

4. Lohman TG, Ring K, Pfeiffer K, Camhi S, Arredondo E, Pratt $C$, et al. Relationships among fitness, body composition, and physical activity. Med Sci Sports Exerc. 2008; 40(6):1163-70. doi: 10.1249/MSS.0 b013e318165c86b.

5. Nelson ME, Rejeski WJ, Blair SN, Duncan PW, Judge JO, King AC, et al. Physical activity and public health in older adults: recommendation from the American College of Sports Medicine and the American Heart Association. Circulation. 2007; 116(9):1094-105. doi: 10.1161/CIRCULATIONAHA. 107.185649 .

6. Carnethon MR, Sternfeld B, Schreiner PJ, Jacobs Jr DR, Lewis CE, Liu K, et al. Association of 20-year changes in cardiorespiratory fitness with incident type 2 diabetes: the coronary artery risk development in young adults (CARDIA) fitness study. Diabetes Care. 2009; 32(7):1284-8. doi: 10.2 337/dc08-1971.

7. Wei M, Kampert JB, Barlow CE, Nichaman MZ, Gibbons LW, Paffinbarger Jr RSP, et al. Relationship between low cardiorespiratory fitness and mortality in normal-weight, overweight, and obese men. JAMA. 1999; 282(16):1547-53. doi: 10.1001/jama.2 82.16.1547.

8. Laukkanen JA, Kurla S, Salonena R, Rauramaa R, Salonen JT. The predictive value of cardiorespiratory fitness for cardiovascular events in men with various risk profiles: a prospective population-based cohort study. Eur Heart J. 2004; 25(16):1428-37. doi: 10.1 016/j.ehj.2004.06.021. 
9. Jurca R, Jackson AS, LaMonte MJ, Morrow Jr. JR, Blair SN, Wareham NJ, et al. Assessing cardiorespiratory fitness without performing exercise testing. Am J Prev Med. 2005; 29(3):185-93. doi: 10.1016/j.amepre.2005.06.004.

10. Carnethon MR, Gulati M, Greenland P. Prevalence and cardiovascular disease correlates of low cardiorespiratory fitness in adolescents and adults. JAMA. 2005; 294(23):2981-8. doi: 10.1001/jama.2 94.23.2981.

11. Hussey J, Bell C, Bennett K, O’Dwyer J, Gormley J. Relationship between the intensity of physical activity, inactivity, cardiorespiratory fitness and body composition in 7-10-year-old Dublin children. $\mathrm{Br}$ J Sports Med. 2007; 41(5):311-6. doi: 10.1136/bjsm.2 006.032045.

12. Bell LM, Watts K, Siafarikas A, Thompson A, Ratnam N, Bulsara $M$, et al. Exercise alone reduces insulin resistance in obese children independently of changes in body composition. J Clin Endocrinol Metab. 2007; 92(11):4230-5. doi: 10.1210/jc.20 07-0779.

13. Vuori IM. Dose-response of physical activity and low back pain, osteoarthritis, and osteoporosis. Med Sci Sports Exerc. 2001; 33(6 Suppl):S551-86; discussion 609-10. doi: 0195-9131/01/3306-0551.

14. FitzGerald SJ, Barlow CE, Kampert JB, Morrow Jr JR, Jackson AW, Blair SN. Muscular fitness and all-cause mortality: prospective observations. J Phys Activ Health. 2004; 1(1):7-18.

15. Hopper D, Deacon S, Das S, Jain A, Riddell D, Hall $\mathrm{T}$, et al. Dynamic soft tissue mobilisation increases hamstring flexibility in healthy male subjects. $\mathrm{Br} \mathrm{J}$ Sports Med. 2005; 39:594-8. doi: 10.1136/bjsm.20 04.011981.

16. Gale CR, Martyn CN, Cooper C, Sayer AA. Grip strength, body composition, and mortality. Int J Epidemiol. 2007; 36(1):228-35. doi: 10.1093/ije/d yl224.

17. Prista A, Maia JA, Damasceno A, Beunen G. Anthropometric indicators of nutritional status: implications for fitness, activity, and health in school-age children and adolescents from Maputo, Mozambique. Am J Clin Nutr. 2003; 77(4):952-9.

18. Shang $X$, Liu A, Li Y, Hu X, Du L, Ma J, et al. The association of weight status with physical fitness among Chinese children. Int J Pediatrics. 2010; 2010(2010):1-6. doi: 10.1155/2010/515414.

19. Huang YC, Malina RM. Physical activity and healthrelated physical fitness in Taiwanese adolescents. J Physiol Anthropol Appl Human Sci. 2002; 21(1):11-9.
20. Vuori I. Health benefits of physical activity with special reference to interaction with diet. Public Health Nutr. 2001; 4(2):517-28. doi: 10.1079/PHN 2001137.

21. Bovet $P$, Auguste $R$, Burdette $H$. Strong inverse association between physical fitness and overweight in adolescents: a large school-based survey. Int J Behav Nutr Phys Act. 2007; 4(24):1-8. doi: 10.1186/1479-5868-4-24.

22. Caspersen CJ, Powell KE, Christenson GM. Physical activity, exercise, and physical fitness: definitions and distinctions for health-related research. Public Health Rep. 1985; 100(2):126-31.

23. Ainsworth BE, Haskell WL, Whitt MC, Irwin ML, Swartz AM, Strath SJ, et al. Compendium of physical activities: an update of activity codes and MET intensities. Med Sci Sports Exerc. 2000; 32(9 Suppl):S498-504. doi: 0195-9131/00/3209-0498/0.

24. World Health Organization. Exercise for health. Bull World Health Organ. 1995; 73(2):135-6. doi: cgi-bin/ wxislind.exe/iah1995/73.

25. Canadian Society for Exercise Physiology Canada's physical activity guideline for children. Minister of Public Works and Government Services Canada. Canada: Health Canada; 2002.

26. Volek JS, Gomez AL, Love DM, Weyers AM, Hesslink $R$, Jr., Wise JA, et al. Effects of an 8-week weightloss program on cardiovascular disease risk factors and regional body composition. Eur J Clin Nutr. 2002; 56(7):585-92. doi: 10.1038/sj.ejcn. 1601362.

27. Lakka TA, Laaksonen DE. Physical activity in prevention and treatment of the metabolic syndrome Appl Physiol Nutr Metab. 2007; 32(1):76-88. doi: 10.1139/H06-113.

28. Hanley AJ, Harris SB, Gittelsohn J, Wolever TM, Saksvig B, Zinman B. Overweight among children and adolescents in a Native Canadian community: prevalence and associated factors. Am J Clin Nutr. 2000; 71(3):693-700.

29. World Health Organization. Physical status: the use and interpretation of anthropometry. Geneva: WHO; 1995. Technical Report Series, 854.

30. Vaz M, Thangam S, Prabhu A, Shetty PS. Maximal voluntary contraction as a functional indicator of adult chronic undernutrition. Br J Nutr. 1996; 76(1): 9-15. doi: 10.1079/BJN19960005.

31. Serrano M, Collazos JFR, Romero SM, Santurino M, Armesilla C, Cerrod JLPd, et al. Handgrip strength in children and teenagers aged from 6 to 18 years: Reference values and relationship with size and body composition. An Pediatr. 2009; 70(4):340-8. doi: 10.1016/j.anpedi.2008.11.025.

32. Paiva MG, Marinho S, Amaral P, Canon F, Barros K, Magalhães de Castro R, et al. Mechanical properties 
of the plantar flexor muscles in malnourished prepubertal children. Comp Method Biomechanics Biomed Eng. 2008; 11(suppl001):179-80. doi: 10.1080/10255 840802298737.

33. Kim J, Must A, Fitzmaurice GM, Gillman MW, Chomitz V, Kramer E, et al. Relationship of physical fitness to prevalence and incidence of overweight among schoolchildren. Obes Res. 2005; 13(7): 1246-54. doi: 10.1038/oby.2005.148.

34. Deforche B, Lefevre J, Bourdeaudhuij ID, Hills AP, Duquet W, Bouckaert J. Physical fitness and physical activity in obese and nonobese Flemish youth. Obes Res. 2003; 11(3):434-41. doi: 10.1038/oby.200 3.59 .

35. Kilbride HW, Gelatt MC, Sabath RJ. Pulmonary function and exercise capacity for ELBW survivors in preadolescence: effect of neonatal chronic lung disease. J Pediatr. 2003; 143(4):488-93. doi: 10.1 067/S0022-3476(03)00413-X.

36. Jensen $C B$, Storgaard $H$, Madsbad S, Richter EA, Vaag AA. Altered skeletal muscle fiber composition and size precede whole-body insulin resistance in young men with low birth weight. J Clin Endocrinol Metab. 2007; 92(4):1530-4. doi: 10.1210/jc.20062360.

37. Hales CN, Barker DJ. The thrifty phenotype hypothesis. Br Med Bull. 2001; 60(1):5-20. doi: 10.1 093/bmb/60.1.5.

38. Kramer MS. Determinants of low birth weight: methodological assessment and meta-analysis. Bull World Health Organ. 1987; 65(5):663-737.

39. Lucas A. Programming by early nutrition in man. Ciba Found Symp. 1991; 156(4):38-50; discussion5. doi: 10.1002/9780470514047.ch4.

40. Lucas A. Long-term programming effects of early nutrition-implications for the preterm infant. J Perinatol. 2005; 25(Suppl 2):S2-6. doi: 10.1038/s j.jp.7211308.

41. Barker DJ. Early growth and cardiovascular disease. Arch Dis Child. 1999; 80(4):305-7. doi: 10.1136/a dc.80.4.305.

42. Rogers M, Fay TB, Whitfield MF, Tomlinson J, Grunau RE. Aerobic capacity, strength, flexibility, and activity level in unimpaired extremely low birth weight $(<800 \mathrm{~g})$ survivors at 17 years of age compared with term-born control subjects. Pediatrics. 2005; 116(1):58-65. doi: 10.1542/peds. 2004-1603.

43. Smith LJ, van Asperen PP, McKay KO, Selvadurai $H$, Fitzgerald DA. Reduced exercise capacity in children born very preterm. Pediatrics. 2008; 122(2): 287-93. doi: 10.1542/peds.2007-3657.

44. Burns YR, Danks M, O'Callaghan MJ, Gray PH, Cooper D, Poulsen L, et al. Motor coordination difficulties and physical fitness of extremely-lowbirthweight children. Dev Med Child Neurol. 2008; 51(2):136-42. doi: 10.1111/j.1469-8749.2008.03 118.x.

45. Kriemler S, Keller H, Saigal S, Bar-Or O. Aerobic and lung performance in premature children with and without chronic lung disease of prematurity. Clin J Sport Med. 2005; 15(5):349-55. doi: 00 042752-200509000-00011.

46. Whitfield MF, Grunau RE. Teenagers born at extremely low birth weight. Paediatr Child Health. 2006; 11(5):275-7.

47. Bass S, Eser P, Daly R. The effect of exercise and nutrition on the mechanostat. J Musculoskelet Neuronal Interact. 2005; 5(3):239-54.

48. Maccari S, Darnaudery M, Morley-Fletcher S, Zuena AR, Cinque C, van Reeth O. Prenatal stress and long-term consequences: implications of glucocorticoid hormones. Neurosci Biobehav Rev. 2003; 27(1-2): 119-27. doi: 10.1016/S0149-7634(03)00014-9.

49. Weinstoc M. Does prenatal stress impair coping and regulation of hypothalamic-pituitary-adrenal axis! Neurosci Biobehavi Rev. 1997; 21(1):1-10.

50. Tong JF, Yan X, Yan X, Ford SP, Nathanielsz PW, Du M. Maternal obesity downregulates myogenesis and $\beta$-catenin signaling in fetal skeletal muscle. Am J Physiol Endocrinol Metab. 2009; 296(4):917-24. doi: 10.1152/ajpendo.90924.2008.

Recebido em: 18/11/2010

Versão final reapresentada em: 5/5/2011 Aprovado em: 21/7/2011 